\title{
Hematogenous muscular metastasis of NSCLC in FDG-PET/CT
}

\author{
Hiroaki Satoh, Tomohiro Tamura, Katsunori Kagohashi
}

Division of Respiratory Medicine, Mito Medical Center, University of Tsukuba, Japan

Contemp Oncol (Pozn) 2015; 19 (5): 422-422

DOI: $10.5114 /$ wo.2015.56009

We read with interest the article by Savas et al. ( $3^{\text {rd }}$ issue, vol. 19, 2015)[1] on hematogenous muscular metastasis of NSCLC in FDG-PET/CT. We would like to ask some queries. First, I would like to know about the definition of "muscle metastasis" used in the article. There might be two kinds of "muscle metastasis": one with direct metastasis to muscle tissue, and other with muscle invasion from adjacent tissue metastasis such as bone, soft connective tissue, and skin. Did the authors included them or not? Could FDG-PET/CT differentiate them? Second, the authors described the size of muscle metastasis: $5-30 \mathrm{~mm}$. How much size could detect it by FDG-PET/CT? Third, the authors described that they confirmed muscle metastasis histopahtologically in three patients. How they obtained it, biopsy or totally resection? How about the relationship with surrounding muscle, invaded or isolated? How the authors confirm it as hematogenous metastasis? Fourth, the authors followed up patients with confirmation of muscle metastasis. We would like to know whether the lesion muscle metastasis respond to chemotherapy or other therapies. If not, the lesions enlarged rapidly or not?

The authors declare no conflict of interest.

\section{Reference}

1. Savas K, Pinar KZ, Sevda KS, Ugur K, Evrim S, Halit C, Sevdegul M, Ansal BT. Haematogenous muscular metastasis of non-small cell lung cancer in F-18 fluorodeoxyglucose positron emission tomography/computed tomography. Contemp Oncol (Pozn) 2015; 19: 241-5.

\section{Address for correspondence}

Hiroaki Satoh MD, PhD

Division of Respiratory Medicine

Mito Medical Center

University of Tsukuba

Miya-machi 3-2-7, Mito

Ibaraki 310-0015, Japan

tel. +81-29-231-2371

e-mail: hirosato@md.tsukuba.ac.jp 